\title{
Expanded Dengue Syndrome: Presenting as Overt Thyrotoxicosis without stigmata of Graves' disease (A Case Report)
}

\author{
${ }^{1}$ Dr Talib S.H, ${ }^{2}$ Dr Sainani Rahul, ${ }^{3}$ Dr Ameet Chordiya \\ ${ }^{1}$ Professor \& Head, ${ }^{2}$ Senior Resident In Medicine, ${ }^{3}$ Seniorresident In Medicine \\ Department of Medicine Mahatma Gandhi Missions Medical College \& Hospital, Aurangabad - 431003
}

\begin{abstract}
We present a case of dengue febrile illness as expanded dengue spectrum, with symptoms of overt hyperthyroidism without ophthalmopathy \& other stigmatas of Graves' disease including antithyroid antibodies. This atypical presentation of non- goitrous, non ophthalmic Graves' disease as an etiology of overt thyrotoxicosis is aided by performing technetium 99 scan study differentiating this atypical Graves disease' from thyroiditis \&/or toxic thyroid nodule.
\end{abstract}

Key words : Dengue fever, Overt thyrotoxicosis, Graves'disease, expanded dengue syndrome.

\section{Introduction:}

Dengue fever is a viral infection transmitted by the mosquito Aedes Aegypti. Aedes Aegypti mosquito is found in tropic \& subtropic regions \& is commonly known to flourish due to unplanned urbanisation \& poor control measures. The symptomatic dengue infection follows an uncomplicated course, however, unusual manifestations of this disease are now being increasingly reported as expanded dengue syndrome which incorporates wide spectrum of uncommon presentation of this common disease.

\section{Case History-}

A 27 year old Indian male patient was referred to our tertiary care hospital who presented with loose stools, high grade fever \& breathlessness for 5 days prior to the hospitalization.His high grade temperature was associated with headache, retro-orbital pain, myalgias, chills, vomiting and weight loss of $3 \mathrm{~kg}$ over last 2 weeks. There were no signs/symptoms of bleeding diathesis.

The patient was a non smoker, non alcoholic and denied any significant familily history including that of thyroid. On examination, patient was moderately dyspneic, with a respiratory rate of $30 / \mathrm{min}, \&$ had a temperature of $39^{\circ} \mathrm{C}$. There was marked tachycardia with a pulse rate of 130/min in sinus rhythm, also had mild signs of dehydration and icterus which were salient findings on examination. Clubbing, lymphadenopathy \& bleeding spots were not noted. He had no palpable organomegaly or masses found except $1 \mathrm{~cm}$ tender hepatic enlargement.Other systems were clinically unremarkable on physical examination.

This patient's lab work up included $\mathrm{Hb} 10.2 \mathrm{gm} \%$, haematocrit 34\%,WBC 15,140/cu.mm ( N 88\%, L $10 \%$,E $01 \%, \mathrm{M} 01 \%$, B $0 \%$ ).Platelets which were initially 1,29,000/cu.mm dropped to 95,000/cu.mm in 2 days but improved in 10 days post-illness. ,Serum sodium \& potassium were within normal limits. Prothrombin time: 13sec , INR-1.22. Sr. bilirubin(T)-5.7mg/dl, Sr. bilirubin(D)-4.6mg/dl,SGOT/AST -34(N1537)IU/L,SGPT/ALT -40(N30-65)IU/L,Sr Alkaline Phosphatase-169 IU/L(N50-136),Total proteins6.2gm/dl(N6.4-8.2),Total albumin-2.6gm/dl(N3.4-5.0) with A:G-0.7. Hepatitis B \& hepatitis C antibodies were negative. The chest X-ray (PA) revealed bilateral minimal pleural effusion \& bilateral patchy pneumonitis. Dengue fever was suspected in this febrile patient .Specific serological tests done for dengue including NS-1 antigen, IgG, IgM antibodies were positive for dengue fever.

Patient was placed on non invasive ventilation for 4 days with supplemental crystalloid IV fluids and beta lactam antibiotics. .His general condition improved in a week, however sinus tachycardia persisted with rate 120-130 beats/min. His clinical examination showed no thyroid masses or nodules or any tenderness. He had mild fine hand tremors \& a staring gaze. The thyroid function tests showed markedly high $\mathrm{FT}_{3}$ of $5.12 \mathrm{pg} / \mathrm{ml}$ $(\mathrm{N}-0.80-1.90), \mathrm{FT}_{4}$ of $10.9 \mathrm{ng} / \mathrm{dl}(\mathrm{N}-1.5-4.10)$, and significantly reduced TSH of $0.004 \mathrm{uIU} / \mathrm{mL}(\mathrm{N}-0.400-$ 4.00). CT scan thyroid revealed no nodules but showed diffuse increased vascularity. ${ }^{99}$ Technetium thyroid scan was performed which showed increased vascularity of both lobes of thyroid with delayed state image indicative of mildly enlarged thyroid (left>right) with diffuse increased radiouptake. No definite hot or cold nodules were seen. The total thyroid uptake was $7.4 \%$ (normal 1 to $4 \%$ ) and the thyroid to parotid ratio was 7.98 (normal 0.9 to $2.9 \%$ ). The values greater than $2.5 \%$ of thyroid to parotid ratio indicate hyperthyroidism. The scan findings were indicative of Graves'disease. Patient was placed on oral Neomercazole and Propanolol besides palliative therapy. He was discharged home after a month of hospitalization. Patient was followed on an outpatient basis by our team at 6 weeks post-hospitalisation with thyroid profile of overt thyrotoxicosis - $\mathrm{FT}_{3}$ of $8.51 \mathrm{pg} / \mathrm{ml}(\mathrm{N}-0.80-1.90), \mathrm{FT}_{4}$ of $2.28 \mathrm{ng} / \mathrm{dl}(\mathrm{N}-1.5-4.10)$, TSH of $0.016 \mathrm{uIU} / \mathrm{mL}(\mathrm{N}-0.400-4.00) \&$ pulse rate 
varied from 120 to 130 beats/min in sinus rhythm. However values though elevated, found receding from previous values. Patient is comfortable and stable.

\section{Discussion:}

Dengue disease and its severity is classified based on WHO classification system $2011^{1}$. Although most symptomatic infections follow an uncomplicated course, complications \& unusual manifestations are now being increasingly recognized. Expanded dengue syndrome has unusual and atypical manifestations which includes various neurological, hepatic, renal, myocardial \& other isolated organ involvements expressed as complications of severe profound shock or associated host conditions or co- infections. We present a case of dengue syndrome with atypical clinical presentations of Graves' disease including anemia ,diarrhoea and jaundice. Graves' disease accounts for up to $80 \%$ of hyperthyroidism cases \& is estimated to affect $0.5 \%$ of population ${ }^{2}$. Anemia has been found in $33 \%$ of Graves'disease patients as presenting feature ${ }^{3}$. The case becomes complex when features of hyperthyroidisms are overlooked,especially in presence of anemia. Graves'disease anaemia may include pernicious anaemia, autoimmune haemolytic anaemia, iron deficiency anaemia or unexplained anaemia associated with Graves' disease ${ }^{4}$. The other atypical manifestation includes presence of jaundice.The spectrum of liver involvement in Graves' disease extends from asymptomatic biochemical abnormality to frank hepatitis, in majority of cases, the presence of biochemical abnormality attracts the physician rather than clinically obvious liver disease ${ }^{\mathbf{5 , 6}}$.

The mechanism of hepatic injury appears to be relative hypoxia due to increase in hepatic oxygen demand without increase in hepatic blood flow . Liver may get damaged by systemic effects of excess thyroid hormones which may have a direct toxic effect on tissues ${ }^{8}$. Lack of knowledge to association these findings with Graves' disease may lead to delay in diagnosis or cause mis diagnosis \&/or unwarranted workup in the case. According to figures included in the academic survey of Maharashtra 2011 - jan 2012 , the dengue cases for the period recorded were 7898 as opposed to 3746 cases the year before'. A large outbreak of dengue fever hit State of Maharashtra, INDIA in September 2012. .As a result of emergence of large no. of cases during the present outbreak of dengue fever, atypical features are now increasingly recognized. The present case has presented with overt thyrotoxicosis ( atypical Graves' disease ) during the course of dengue febrile illness ${ }^{1}$.Findings of marked suppression of TSH $0.004 \mathrm{uIU} / \mathrm{mL}(\mathrm{N}-0.400-4.00)$ with elevated FT3 \& FT4, increased diffuse tracer uptake \& increased vascularity by thyroid gland of both lobes on immediate ${ }^{99 \mathrm{~m}} \mathrm{Tc}\left(\mathrm{O}^{4--}\right)$ thyroid scintigraphy flow images suggest overt thyrotoxicosis which differentiates Graves' disease from transient thyroiditis. The presence of raised serum concentration of thyroperoxidase ( TPO ) antibodies indicate an autoimmune thyroid disorder \& a raised TSI value indicates Graves' disease. Treatment of overt thyrotoxicosis is much based on imperical evidence ; to date relatively few large scale randomized clinical trials have been conducted. For this reason treatment preferences vary substantially by region ${ }^{10}$. In the present case TPO antibodies were negative \& the estimation of Sr TSI could not be undertaken because of patient's financial constraints. Differentiating between subacute transient thyroiditis \& Graves' disease is essential, as medical treatment for hyperthyroidism is often not needed for transient thyroiditis because symptoms of transient thyroiditis are shortlived. However, beta blockers may prove beneficial for symptomatic relief. Assir HZK \& associates in Sep 2012 reported 1st case of thyroiditis with dengue fever in the medical literature from Pakistan ${ }^{11}$. To the best of our knowledge, we humbly claim that overt thyrotoxicosis with atypical features of Graves'disease developing during the course of dengue fever has not been previously reported in the literature \& should be included as a manifestation of expanded dengue syndrome.

\section{Conclusion :}

Overt hyperthyroidism with non-stigmata Graves' disease may develop during the course of dengue fever. Unexplained tachyarrhythmias, jaundice, anemia, increased bowel movements in patients with dengue fever with or without thyroid enlargement guard us to further investigate the case for evidence of hyperthyroidism. Differentiating transient thyroiditis ( subacute ) from Graves' disease is essential for advocation \& placement of appropriate medical therapy.

\section{References}

[1]. WHO: Comprehensive guidelines for prevention and control of dengue and dengue hemorrhagic fever. World Health Organization, Regional Office for South-East Asia; 2011.

[2]. Brent GA. Clinical practice Graves' disease. NEJM.2008; $358: 2594-05$.

[3]. Hegazi M O and Ahmed S. Atypical Clinical Manifestations of Graves' Disease:An Analysis in Depth. Journal of Thyroid Research. 2012; Article ID 768019.

[4]. Gianoukakis A G, Leigh M J, Richards P et al. Characterization of the anaemia associated with Graves' disease.Clinical Endocrinology. 2009;70 ( 5 ): 781-87.

[5]. Hegazi M O and El-sonbaty M R. Unusual presentations of hyperthyroidism. Handbook of Hyperthyroidism: Etiology,Diagnosis and Treatment.2011;265-70.

[6]. Maheshwari A and Thuluvath P J. Endocrine diseases and the liver. Clinics in Liver Disease.2011;15: 55-67. 
[7]. Malik R and Hodgson H. The relationship between the thyroid gland and the liver.QJM.2002;95:559-69.

[8]. Owen P J, Baghomian A, Lazarus J H, and Godkin A J. An unusual cause of jaundice. BMJ.2007;335: 773-74

[9]. Dengue cases double, deaths rise four times.Published: Sunday, Mar 25 , 8:00 IST.By Rito Paul /Place:Mumbai/Agency:DNA http://www.dnaindia.com/mumbai/report_dengue-cases-double-deaths-rise-four-times_1667057

[10]. Pearce EN. Clinical Review.Diagnosis and management of thyrotoxicosis.BMJ.2006;332:1369-73.

[11]. Assir MZK, Jawa A , and Ahmed H I. Expanded dengue syndrome : subacute thyroiditis and intracerebral hemorrhage.BMC Infectious Diseases.2012;12:240. http://www.biomedcentral.com/1471-2334/12/240 